Aim of the study: A recent breast cancer genome-wide association study (GWAS) identified single-nucleotide polymorphism (SNP) rs2046210 on 6q25.1 showing a strong association with breast cancer risk. Numerous association studies have been conducted to investigate the relationship between this polymorphism and breast cancer risk in various populations. There have been conflicting reports about the association of this locus with breast cancer risk in different ethnic groups. For the first time, this study has investigated the association of rs2046210 SNP with breast cancer risk in Iranian Azari-Turkish women in North West Iran.

Material and methods: In this study 192 breast cancer subjects and 186 healthy controls were genotyped using Taqman SNP genotyping assays for different SNP rs2046210 alleles. Results: No significant association between rs2046210 SNP alleles and the risk of breast cancer was detected in Iranian Azari-Turkish women.

Conclusions: The data suggests that rs2046210SNP does not play a role in the aetiology of breast cancer in the Iranian Azari-Turkish population, and it indicates possible genetic differences for breast cancer between different population ancestries. Our result is an important contribution to the literature about genetic susceptibility for breast cancer in Asian populations. Additional studies are required to confirm our findings.

Key words: breast cancer, genetic factors, rs2046210 SNP, Azari-Turkish population.

Contemp Oncol (Pozn) 2016; 20 (4): 308-310 DOI: $10.5114 /$ wo. 2016.6185

\section{Evaluation of a newly discovered breast cancer susceptibility locus at 6q25.1 in Iranian Azari-Turkish women}

\author{
Ziba Garehdaghchi ${ }^{1}$, Siam Mansoori Derakhshan ${ }^{2}$, \\ Mahmoud Shekari Khaniani²
}

${ }^{1}$ Department of Biology, Faculty of Basic Sciences, Islamic Azad University Ahar Branch, Ahar, Iran

${ }^{2}$ Department of Medical Genetics, School of Medicine, Tabriz University of Medical Sciences, Tabriz, Iran

\section{Introduction}

Breast cancer is one of the most common malignancies affecting women in Iran, as in many other parts of the world. Breast cancer is a multifactorial disease resulting from different environmental and complex polygenic risk factors playing an extensive role in the aetiology of the disease [1]. Up to approximately $30 \%$ of breast cancer cases may be caused by genetic factors [2]. Several studies have strongly reported the association between genetic factors and both sporadic and familial breast cancer, specially BRCA1 and BRCA2 genes [2]. Family history of breast cancer is a major risk, but the high-penetrant genes that have been presented, such as BRCA1, BRCA2, PTEN, and p53, only explain $20 \%$ to $25 \%$ of familial breast cancer and $5 \%$ of all breast cancers [3].

Recently, several GWAS of breast cancer have identified the association of a number of genetic variants and breast cancer susceptibility [4, 5]. The reported GWAS have mostly been documented within European and Chinese ancestries [6, 7]. In a GWAS of breast cancer in Chinese women a new SNP, rs2046210, was reported to have a strong association with breast cancer risk $[8,9]$. As allele frequencies of SNPs are highly variable and the linkage disequilibrium patterns differ between ethnicities, it is important to confirm this breast cancer susceptibility in different ethnic populations. Therefore, a number of studies have been conducted in various populations, and there have been discrepancies between reports about the association of this locus with breast cancer risk in different ethnic populations. This association was confirmed in European and East-Asian populations, but no association was present in women of African ancestry [10-12]. Additionally, another study reported that SNP rs2046210 may play a role in the aetiology of endometrial cancer in Chinese women [4]. The North West population in Iran with AzeriTurkish ethnicity is genetically distinct from other Iranian ethnic groups. For the first time, this study has investigated the association of this locus with breast cancer risk in Iranian Azari-Turkish women in North West Iran.

\section{Material and methods}

\section{Subjects}

In this study, 192 women with breast cancer participated who were 30 to 69 years of age and permanent residents of North West Iran and were previously diagnosed with breast cancer from two different hospitals (Al Zahra and Taleghani Hospitals, Tabriz University of Medical Sciences) between 2011 and 2012. The diagnosis of breast cancer was based on standard clinical, mam- 
mographic, radiological, and histological criteria. The study also randomly included 186 ethnically sex-matched healthy controls without cancer diseases, who were unknown to each other or even to the patients. Meanwhile, there was no breast cancer in first-degree relatives and age over 40 years. This study was approved by the ethics committees (Ethics Committee of Tabriz University of Medical Sciences), and all patients signed consent forms to participate in this study.

\section{Molecular analysis}

About $5 \mathrm{ml}$ of intravenous blood samples were collected in EDTA Vacutainers, and genomic DNA was prepared by the standard phenol-chloroform procedure $\left(^{*}\right)$. Genotyping was performed using the TaqMan allelic discrimination procedure under standard conditions according to the manufacturer's instructions (Applied Biosystems, Marcogen Company, Seoul, South Korea). Laboratory methods were previously published [4]. In brief, each assay was carried out using $10 \mathrm{ng}$ genomic DNA in a $5 \mu \mathrm{l}$ reaction using Taqman Universal PCR Master Mix (ABI), forward and reverse primers, and FAM- and VIC-labelled probes purchased from Applied Biosystems (ABI Pre-Designed assays). For quality control, 46 random samples were genotyped in duplicate and had identical genotyping assignments. Investigators were blinded as to whether samples were duplicates, cases, or controls.

\section{Statistical analysis}

Deviations of the genotype frequencies in the controls from those expected under Hardy-Weinberg equilibrium were evaluated by $\chi^{2}$ test (DF $=1$ ). Breast cancer risk associated with rs2046210 was estimated as odds ratios $(\mathrm{OR})$ and $95 \%$ confidence intervals $(95 \% \mathrm{Cl})$ using unconditional logistic regression with multiple genetic models, including the genotype model (separate indicators for heterozygotes and rare homozygotes), dominant model (indicator for heterozygotes and rare homozygotes combined), recessive model (indicator for homozygotes), and log additive (per-allele) model (each copy of rare allele) with the common homozygote as the reference category. For all the analyses, differences were considered statistically significant when the $P$ value was less than or equal to 0.05. Genotype and allele associations were calculated using the SPSS 18.0 software package.

\section{Results}

No significant association between rs2046210 SNP and the risk of breast cancer was detected in North West Iran. The frequency distributions of rs2046210alleles are shown in Table 1. The comparison of allele distribution using $\chi^{2}$ test demonstrated no significant association between the two groups $(p>0.05)$. For more details, the frequency recordings of homozygous and heterozygous alleles were performed separately. Most of the alleles in patients and control cases were heterozygote, and $A / G$ allele was the most frequent in both groups. Also, the frequencies of $\mathrm{G} / \mathrm{G}$ and A/A homozygous alleles were the most frequently recorded alleles, respectively, after the same in two $A / G$ allele group (Table 2).

\section{Discussion}

Breast cancer is one of the most common malignancies diagnosed among women in Iran, as in other parts of the world [13]. The incidence of breast cancer has risen in many Asian countries in the past few decades [13, 14]. This increased incidence trend may continue with social style changes. Therefore, early recognition, in order to improve breast cancer consequences and survival, is needed. One of the alternative approaches to prevent this disorder is to identify genetically high-risk women.

Breast cancer has a complex pathogenesis and genetic factors are essential characters in sporadic and familial breast cancer. However, only a small portion of breast can-

Table 1. Age at diagnosis, histology results, and ethnicity breakdown of breast cancer cases and controls in the study

\begin{tabular}{|ccc|}
\hline & Case & Control \\
Age (year) & & \\
$>30$ & 22 & 0 \\
$31-40$ & 78 & 0 \\
$41-50$ & 92 & 9 \\
$51-60$ & 0 & 51 \\
$>61$ & 0 & 126 \\
\hline Total & 192 & 186 \\
\hline Histology & $180(95 \%)$ & - \\
\hline Invasive ductal cancer & $12(5 \%)$ & - \\
\hline Invasive lobular cancer & Azari-Turkish & Azari-Turkish \\
\hline Race &
\end{tabular}

Table 2. Population structure of breast cancer cases and controls with rs2046210 allele and genotype frequencies used in the study

\begin{tabular}{|c|c|c|c|c|}
\hline & Patients $(n=192)$ & Controls $(n=186)$ & OR $(95 \% \mathrm{Cl})$ & $P$ value \\
\hline $\mathrm{A} / \mathrm{A}$ & 31 (16.1\%) & 33 (17.7\%) & 1 & \\
\hline $\mathrm{A} / \mathrm{G}$ & $105(54.7 \%)$ & $95(51.1 \%)$ & $0.85(0.48-1.49)$ & 0.57 \\
\hline $\mathrm{G} / \mathrm{G}$ & $56(29.2 \%)$ & $58(31.2 \%)$ & $0.97(0.52-1.79)$ & 0.93 \\
\hline \multicolumn{5}{|l|}{$2 \mathrm{df}$ test } \\
\hline Dominant & & & $0.86(0.57-1.29)$ & 0.48 \\
\hline Recessive & & & $0.92(0.61-1.24)$ & 0.38 \\
\hline Per allele & & & $0.88(0.45-1.02)$ & 0.12 \\
\hline
\end{tabular}


cer subjects can be explained by identified susceptibility genes, such as BRCA1 and BRCA2.

Considering this problem, a number of GWAS of breast cancer have reported some susceptibility genetic variant with breast cancer in European, Asian ancestries [15] because positive candidate genes with an association to breast cancer in individuals of an ethnic group may be negative in another ethnic population. It is important to understand the effects of the GWAS-discovered markers in women of other ethnicities. In the Asian population, in Korean and Chinese women a new genetic variant rs2046210SNP associated with breast cancer has recently been identified. This SNP is located approximately $29 \mathrm{~kb}$ upstream of the ESR1 gene. Because of its relative vicinity to the ESR1 gene, it is possible that SNP rs2046210 effects ESR1 gene function and thereby cause susceptibility to breast cancer [5].

In a pooled analysis of women of East-Asian, European, and African ancestry, positive association for rs2046210 and breast cancer risk in Chinese women [ORs (95\% Cl) $1 / 41.30(1.22-1.38)$ and 1.64 (1.50-1.80) for the AG and AA genotypes, respectively, $p$ for trend $1 / 4 \quad 1.54 \quad 10 \quad 30]$, European-ancestry American women [ORs $(95 \% \mathrm{CI})^{1 / 4} 1.07$ (0.99-1.16) and 1.18 (1.04-1.34), $p$ for trend $1 / 40.0069]$, and Japanese women [ORs (95\% CI)1/4 1.31 (1.13-1.52) and 1.37 (1.06-1.76), $p$ for trend $\left.1 / 42.51 \_10 \_4\right]$ was found. No association with this SNP was observed in African American women [ORs $(95 \% \mathrm{CI}) 1 / 40.81(0.63-1.06)$ and 0.85 (0.651.11) for the $A G$ and $A A$ genotypes, respectively, $p$ for trend $1 / 4$ 0.4027].

To our knowledge, this is the first study in an Iranian Azari-Turkish population that has evaluated breast cancer susceptibility rs2046210SNP locus. Here, we report for the first time that rs2046210 SNP identified from earlier GWAS was not associated with breast cancer in our population.

Our study provides evidence against the hypothesis that rs2046210 polymorphisms are associated with breast cancer risk. However, our results do not rule strongly previous studies from other ethnicities, and additional studies are needed to confirm our findings.

In conclusion, this case-control study involving 378 participants with and without breast cancer showed that the rs2046210 SNP was not significantly associated with breast cancer susceptibility in Iranian Azari Turkish women in North West Iran.

The results from our study are an important contribution to the literature about genetic susceptibility for breast cancer in Asian populations.

We would like to thank Dr M. Ghojazadeh from the Research Development \& Coordination Centre (RDCC) of Tabriz University of Medical Sciences for reviewing the statistical analysis. Control samples were provided by Ebbne Sina Prenatal Diagnostic and Medical Genetics Centre Tabriz University of Medical Sciences, Tabriz, Iran.

The authors declare no conflict of interest.

\section{References}

1. Long J, Shu XO, Cai Q, et al. Evaluation of breast cancer susceptibility loci in Chinese women. Cancer Epidemiol Biomarkers Prev 2010; 19: 2357-65.

2. Ford D, Easton DF, Stratton M, et al. Genetic heterogeneity and penetrance analysis of the BRCA1 and BRCA2 genes in breast cancer families. The Breast Cancer Linkage Consortium. Am J Hum Genet 1998; 62: 676-89.

3. Gaudet MM, Kirchhoff T, Green T, et al. Common genetic variants and modification of penetrance of BRCA2-associated breast cancer. PLoS Genet 2010; 6: e1001183.

4. Hein R, Maranian M, Hopper JL, et al. Comparison of $6 q 25$ breast cancer hits from Asian and European Genome Wide Association Studies in the Breast Cancer Association Consortium (BCAC). PLoS One 2012; 7: e42380.

5. Stacey SN, Sulem P, Zanon C, et al. Ancestry-shift refinement mapping of the C6orf97-ESR1 breast cancer susceptibility locus. PLoS Genet 2010; 6: e1001029.

6. Zheng W, Long J, Gao YT, et al. Genome-wide association study identifies a new breast cancer susceptibility locus at $6 q 25.1$. Nat Genet 2009; 41: 324-8.

7. Cai Q, Long J, Lu W, et al. Genome-wide association study identifies breast cancer risk variant at 10q21.2: results from the Asia Breast Cancer Consortium. Hum Mol Genet 2011; 20: 4991-9.

8. Zhang XJ, Huang W, Yang S, et al. Psoriasis genome-wide association study identifies susceptibility variants within LCE gene cluster at 1q21. Nat Genet 2009; 41: 205-10.

9. Yang M, Wu Y, Lu Y, et al. Genome-wide scan identifies variant in TNFSF13 associated with serum IgM in a healthy Chinese male population. PLoS One 2012; 7: e47990.

10. Haiman CA, Chen GK, Vachon CM, et al. A common variant at the TERT-CLPTM1L locus is associated with estrogen receptor-negative breast cancer. Nat Genet 2011; 43: 1210-4.

11. Stevens KN, Vachon CM, Lee AM, et al. Common breast cancer susceptibility loci are associated with triple-negative breast cancer. Cancer Res 2011; 71: 6240-9.

12. Stevens KN, Wang X, Fredericksen Z, Pankratz VS, Cerhan J, Vachon CM, Olson JE, Couch FJ. Evaluation of associations between common variation in mitotic regulatory pathways and risk of overall and high grade breast cancer. Breast Cancer Res Treat 2011; 129: 617-22.

13. Yassaee VR, Zeinali S, Harirchi I, Jarvandi S, Mohagheghi MA, Hornby DP, Dalton A. Novel mutations in the BRCA1 and BRCA2 genes in Iranian women with early-onset breast cancer. Breast Cancer Res 2002; 4: R6.

14. Michailidou K, Hall P, Gonzalez-Neira A, et al. Large-scale genotyping identifies 41 new loci associated with breast cancer risk. Nat Genet 2013; 45: 353-61.

15. Cai Q, Wen W, Qu S, et al. Replication and functional genomic analyses of the breast cancer susceptibility locus at $6 q 25.1$ generalize its importance in women of chinese, Japanese, and European ancestry. Cancer Res 2011; 71: 1344-55.

\section{Address for correspondence}

Dr Mahmoud Shekari Khaniani MD, PhD

Department of Medical Genetics

School of Medicine

Tabriz University of Medical Sciences

Tabriz, Iran

tel. +98(41) 33375540

fax $+98(41) 33375540$

e-mail: Mahmoud. khaniani@gmail.com

shekarim@tbzmed.ac.ir

Submitted: 15.12 .2014

Accepted: 15.10 .2015 